(Aus dem pathologischen Institut [Prof. Dr. M. Borst] und der Universitätskinderklinik [Prof. Dr. M. v. Pfa undler] der Universität München.)

\title{
Untersuchungen über den Cholesteringehalt von Frauen- und Kuhmilch.
}

\author{
Von \\ Prof. Dr. L. Wacker, \\ Vorst. der chem. Abtlg. des path. Inst. \\ und \\ Dr. Karl F. Beck, \\ Volontärassistent der Klinik.
}

(Eingegangen am 9. November 1920.)

Gelegentlich unserer Untersuchungen über den Fett- und Cholesterinstoffwechsel bei Säuglingen hatten wir eine größere Anzahl von Milchproben auf ihren Cholesteringehalt zu untersuchen.

In der Literatur finden wir nur sehr spärliche Angaben über diesen Gegenstand, die noch dazu aus einer Zeit stammen, als die von Wind a us angegebene Methode der Cholesterinbestimmung durch Wägen der Digitoninverbindung noch nicht bekannt war. Wir finden in den heute maßgebenden-Werken (Literaturverzeichnis Nr. 2, 3, 4) die von Tolmatscheff angegebenen Werte von 0,25 -0,38 g Cholesterin im Liter Frauenmilch.

Unsere Untersuchungen ergaben, daß nur etwa die Hälfte der Tolmatscheffschen Werte den Tatsachen entspricht, da $\beta$ auch die von Fränkel angegebenen Zahlen nicht den wahren Cholesteringehalt der Milch wiedergeben; dagegen decken sich die neuerdings von Be u mer veröffentlichten 2 Werte gut mit unseren Befunden. Die von Tolmatscheff angegebenen hohen Werte dürften darauf zurückzuführen sein, daß das Unverseifbare des Milchfettes, ebenso wie das anderer Fette, einige hartnäckige Beimengungen enthält, die mit Digitonin nicht fällbar sind, also einer anderen chemischen Körperklasse angehören.

Wir wandten folgende Methodik an: $100 \mathrm{ccm}$ Milch werden mit $2 \mathrm{com} 40$ proz. $\mathrm{KOH}$ versetzt und im Scheidetrichter mit mindestens dem gleichen Volumen Äther $8 \mathrm{mal}$ ausgeschüttelt. Der Äther wird jedesmal mit einer geringen Menge Wassers zur Entfernung allenfalls gelöster Lauge gewaschen und das Waschwasser wieder zu dem auszuäthernden Gut gegeben. In den Äther geht das Fett und das Gesamtcholesterin. Nach Abdampfen des Äthers wird dieser Rückstand im kochenden Wasserbad unter Zugabe geringer Mengen absoluten Alkohols 
und Absaugen der Alkoholdämpfe gründlich getrocknet und zur Feststellung. des Fettgehaltes der Milch gewogen.

Dann wird zur Bestimmung des Gesamtcholesteringehaltes übergegangen. (Die Frage, ob freies und Estercholesterin vorhanden ist, wurde später gesondert untersucht.) Das Fett wird mit $10-20 \mathrm{ccm}$ Alkohol versetzt und dazu $5 \mathrm{ccm} 60 \mathrm{proz}$. $\mathrm{KOH}$ gegeben. Man kocht dann zwecks Verseifung $1 / 2$ Stunde am Rückflußkühler. Nach dieser Zit verdünnt man die Seifenlösung mit Wasser und extrahiert 4 bis 5 mal mit Äther im Scheidetrichter. Der nach dem Verdampfen des Äthers verbleibende, getrocknete Rückstand wird mit $10 \mathrm{ccm}$ absolutem Alkohol versetzt und mit dem thberschuß einer Auflösung von metallischem Natrium ${ }^{1}$ ) in absolutem Alkohol abermals am Rückflußkühler 1 Stunde erhitzt. Nach Abdampfen des überschüssigen Alkohols im kochenden Wasserbad wird mit etwas Wasser verdünnt und 4-5 mal mit Äther ausgeschüttelt. Auch hier wird der Äther jeweils mit Wasser gewaschen, das Waschwasser kommt wieder zur Seifenlösung und wird erneut mit ausgeäthert.

Der Ätherextrakt wird verdampft und der Rückstand nach dem Trocknen nochmals mit möglichst wasserfreiem Äther umgelöst, wobei die in Lösung gegangenen Seifen ungelöst zurückbleiben und abfiltriert werden können. Filter' und Rückstand müssen natürlich mit Äther gut nachgewaschen werden.

Der das Unverseifbare enthaltende Äther wird in einem vorher gewogenen Kölbchen abdestilliert und die Gewichtszunahme des Kölb- . chens nach dem Trocknen des Rückstandes ermittelt. Dieser Rückstand enthält, wie schon erwähnt, außer Cholesterin noch eine Begleitsubstanz, über deren chemische Natur Unklarheit besteht. Die Möglichkeit, daß es sich um geringe Seifenmengen handelt, ist ausgeschlossen, weil beim nochmaligen Umlösen mit Äther oder Petroläther kein Rückstand mehr bleibt, andererseits kann auch kein unverseift gebliebener Cholesterinester mehr vorliegen, denn zur Sicherstellung dieser Verhältnisse wurde in einigen Fällen nochmals mehrere Stunden mit Natriumalkoholat gekocht. Das Vorhandensein von Cholesterinestern würde sich auch an der Schwerlöslichkeit des Rückstandes in Alkohol bei der nachfolgenden Cholesterinbestimmung nach Wind a us erkennen lassen. Man muß also annehmen, daß sich im Unverseifbaren des Fettes noch eine Begleitsubstanz - vielleicht in Form eines anderen hochmolekularen Alkohols - befindet. Im Gegensatz zum Cholesterin besitzt die Begleitsubstanz nur eine geringe Krystallisationsfähigkeit und ist mit Digitonin nicht fällbar.

Zur Klärung der Frage, ob das Cholesterin in der Milch in freier

1) Das Natrium wird zur Entfernung anhaftenden Steinöls mit Äther ge: waschen. 


\begin{tabular}{|c|c|c|c|c|c|c|c|c|}
\hline \multirow[b]{2}{*}{$\begin{array}{c}\begin{array}{c}\text { Datum } \\
\text { der } \\
\text { Untersuchung }\end{array}\end{array}$} & \multirow[b]{2}{*}{$\begin{array}{l}\text { Art der } \\
\text { untersuchten } \\
\text { Milch }\end{array}$} & \multirow[b]{2}{*}{$\begin{array}{l}\text { Fett- } \\
\text { gohalt }\end{array}$} & \multirow[b]{2}{*}{$\begin{array}{l}\text { Chole- } \\
\text { sterin- } \\
\text { gehalt }\end{array}$} & \multirow[b]{2}{*}{$\begin{array}{c}\text { Chole- } \\
\text { sterin- } \\
\text { gehalt } \\
\text { des } \\
\text { Fettes } \\
\% \\
\end{array}$} & \multirow[b]{2}{*}{$\begin{array}{l}\text { Dauer der } \\
\text { Laktation }\end{array}$} & \multicolumn{3}{|c|}{ Durchschnittswerte } \\
\hline & & & & & & $\begin{array}{c}\text { Fett- } \\
\text { gehalt } \\
\% \\
\end{array}$ & $\begin{array}{c}\text { Chole- } \\
\text { sterin- } \\
\text { gehalt } \\
\% \%\end{array}$ & $\begin{array}{c}\text { Chol.- } \\
\text { Gehait } \\
\text { des } \\
\text { Fettes } \\
\% \\
\end{array}$ \\
\hline 29. IV. 20 & Kolostrum & 3,34 & 0,3620 & 1,084 & & 3,34 & 0,3620 & 1,084 \\
\hline 12. VII. 20 & Ammenmilch & 3,33 & 0,1492 & 0,448 & $14 \mathrm{Tg}$. & & & \\
\hline 13. VII. 20 & Edlinger & 3,73 & 0,1515 & 0,406 & 15, & 250 & & \\
\hline 14. VII. 20 & , & 3,42 & 0,1449 & 0,422 & 16 & 3,59 & 0,1448 & 0,415 \\
\hline 15. VII. 20 & $"$ & 3,89 & 0,1339 & 0,383 & 17, & & & \\
\hline 12. XI. 19 & Brustmilch & 2,33 & 0,1917 & 0,823 & $14 .$, & 2,33 & 0,1917 & 0,823 \\
\hline 5. XII. 19 & Markhof & 2,64 & 0,1463 & 0,554 & 37, & & & \\
\hline 7. XII. 19 & " & 2,68 & 0,1477 & 0,550 & 39, & 2,66 & 0,1690 & 0,552 \\
\hline 26. IV. 20 & Ammenmilch & 3,67 & 0,1637 & 0,446 & $44 \%$ & & & \\
\hline 27. IV. 20 & Goldbrunner & 3,48 & 0,1475 & 0,422 & 45, & 3,51 & 0,1577 & $0, \mathbf{4 4 8}$ \\
\hline 28. IV. 20 & 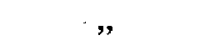 & 3,39 & 0,1620 & 0,477 & 46, & & & \\
\hline 15. X. 19 & Mischmilch & - & 0,1510 & - & ca. 7 Mon. & 366 & & 0405 \\
\hline 24. X. 19 & , & 3,66 & 0,1550 & 0,425 & $\Rightarrow 7$ & & & 0,120 \\
\hline 1. XII. 19 &, & 2,98 & 0,1115 & 0,374 & " 9 & & & \\
\hline 9. XII. 19 & , & 3,53 & 0,1350 & 0,382 & $\Rightarrow \quad 91 / 3$, & & & \\
\hline 10. XII. 19 & , & 3,48 & 0,1214 & 0,348 &, $9^{1 / 3}$, & 3,23 & 0,1096 & 0,336 \\
\hline 11. XII. 19 & ; & 2,95 & 0,0705 & 0,239 & $\Rightarrow 91 / 3$, & & & \\
\hline 17. XII. 19 & , & 4,59 & 0,1180 & 0,257 & $" 91 / 2$, & & & \\
\hline 18. XII. 19 &, & 4,33 & 0,1537 & 0,355 & $\Rightarrow \quad 9^{1 / 2}$, & 4,46 & 0,1358 & 0,306 \\
\hline 11. I. 20 & , & 3,35 & 0,1118 & 0,334 & $\Rightarrow 10^{1} / 2 \Rightarrow$ & & & : \\
\hline 12. I. 20 & , & 3,08 & 0,0963 & 0,312 &, $10^{1 / 2}$ & 3,30 & 0,1114 & 0,336 \\
\hline 13. I. 20 & " & 3,47 & 0,1260 & 0,363 &, $10^{1} / 2$ & & & \\
\hline 16. II. 20 &, & 2,07 & 0,0736 & 0,354 &, $11^{1 / 2}$ & & & \\
\hline 17. II. 20 & , & 1,86 & 0,0667 & 0,356 & $" 11 \frac{1}{2}$ & & & \\
\hline 18. II. 20 & , & 4,09 & 0,1195 & 0,292 &, $11^{1 / 2}$ & 900 & 01046 & $0 \cdot 260$ \\
\hline 19. II. 20 & , & 3,00 & 0,1100 & 0,366 &, $11^{1 / 2}$ & 2,32 & 0,1040 & 0,5000 \\
\hline 20. II. 20 & , & 3,72 & 0,1425 & 0,383 & $" 11^{1 / 2}$, & & & \\
\hline 21. II. 20 & , & 2,81 & 0,1155 & 0,411 &, $11^{1 / 2}$ & & & \\
\hline Durchseh & ttswert aller $u$ & ntersucl & iten Fra & denmile & hproben & 3,29 & 0,1385 & 0,424 \\
\hline 17. X. 19 & Kuhmilch & 3,75 & 0,1590 & 0,424 & & & & \\
\hline 23. X. 19 & m. & 4,00 & 0,1408 & 0,352 & & & & \\
\hline 16. II. 20 & , & 3,31 & 0,1090 & 0,329 & & & & \\
\hline 17. II. 20 & , & 3,46 & 0,1202 & 0,347 & & & & 034 \\
\hline 18. II. 20 & ," & 4,02 & 0,1410 & 0,351 & & 3,00 & 0,1238 & 0,343 \\
\hline 19. II. 20 & , & 3,72 & 0,1175 & 0,315 & & & & \\
\hline 20. II. 20 & , & 3,69 & 0,1110 & 0,300 & & & & \\
\hline 21. II. $20^{\circ}$ & , & 3,31 & 0,1085 & 0,327 & & & & \\
\hline 4. XI. 19 & Magermilch & 0,29 & 0,0234 & 0,807 & & & & \\
\hline 4. XI. 19 & Zentrifugen- & & & & Durchsehn & ttswe & dieser & Milch \\
\hline & & 28,58 & 0,8786 & 0,307 & & 3,98 & & 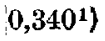 \\
\hline 19. XI. 19 & EiweiBmilch & 4,93 & 0,1870 & 0,379 & \multirow{2}{*}{\multicolumn{4}{|c|}{ (Zwei verschiedene Sendungen) }} \\
\hline 9. XII. 19 & unverdünnt & 4,72 & 0,1810 & 0,383 & & & & \\
\hline
\end{tabular}

1) Es wurden $2140 \mathrm{ccm}$ Vollmilch zentrifugiert: $1860 \mathrm{ccm}$ Magermilch, $280 \mathrm{ccm}$ Rahm. 
oder in Esterform vorhanden ist, wurde erwärmtes Milchfett mit heißem Alkohol wiederholt im Scheidetrichter ausgeschüttelt. Da sich freies Cholesterin leicht in heißem Alkohol löst, müßte es sich beim Erkalten des Alkohols in den charakteristischen Krystallen ausscheiden und außerdem durch Digitonin nachzuweisen sein. Der Nachweis von freiem Cholesterin in der Milch ist uns nicht gelungen, die nachgewiesenen Cholesterinmengen sind als Ester vorhanden. Diese Befunde bestätigen die Beumerschen Angaben.

In der nachstehenden Tabelle sind unsere Untersuchungsergebnisse zusammengestellt:

Es ergibt sich aus der Tabelle, daß die Kolostralmilch den höchsten Cholesteringehalt hat. Eine Abhängigkeit des Cholesteringehaltes von der Dauer der Lactation ist deutlich nachweisbar. Ferner sind individuelle Schwankungen bemerkenswert. So hat die Milch Edlinger einen niedrigeren Cholesteringehalt als die Milch Markhof bei gleich langer Lactation, und als die Milch Markhof und Goldbrunner aus einer späteren Lactationsperiode.

Die Ammenmischmilch aus dem 7. bis 11. Lactationsmonat schwankt ebenfalls in ihrem Cholesteringehalt. Da Cholesterin- und Fettgehalt annähernd parallel gehen, nehmen wir hier Ernährungseinflüsse (fett- bzw. cholesterinarme Nahrung) an. Dies deckt sich auch mit den Befunden Rosenthals, der nachgewiesen hat, daß der Cholesteringehalt des Blutes unter dem Einfluß fettarmer Ernährung während des Krieges abgenommen hat.

Bei den Untersuchungen von 8 -Sorten Kuhmilch ergab sich bei einem durchschnittlich etwas höheren Fettgehalt ein etwas niedrigerer Cholesteringehalt gegenüber der Frauenmilch.

Um den Cholesteringehalt von Magermilch und Rahm festzustellen, wurden $2140 \mathrm{ccm}$ Vollmilch zentrifugiert. Man erhielt $1860 \mathrm{ccm}$ Magermilch und $280 \mathrm{ccm}$ Rahra. Es ergab sich die interessante Tatsache, daß in der Magermilch verhältnismäßig viel Cholesterin enthalten war. Man bekam, wenn man Fett- und Cholesteringehalt zueinander in Beziehung setzte, einen Cholesteringehalt des Fettes von 0,807\%. Die entsprechenden Werte im Rahm und in der Vollmilch sind $0,307 \%$ und $0,343 \%$.

Eine Erklärung dieses auffallenden Befundes dürfte durch die Tatsache, daß das Cholesterin in der Milch nur als Fettsäureester vorhanden ist, gegeben sein. Dieser Ester dürfte zum Teil kolloid in der Molke, zum anderen Teil mit dem Fett zusammen in der Milch vorhanden sein.

\section{Zusammenfassung:}

Der Cholesteringehalt der Frauenmilch ist abhängig von der Dauer der Lactation. Er ist individuellen Schwankungen unterworfen. Die 
292 L. Wacker u. K. F. Beck: Untersuchungen uber den Cholesteringehalt usw.

Ernährung der Stillenden scheint auf den Cholesteringehalt der Milch von EinfluB zu sein.

Durohschnittswert von 28 Frauenmilchanalysen: $3,29 \%$ Fett, $0,1385 \%$ Cholesterin.

." " 8 Kuhmilchanalysen: 3,65, " 0,1258 , "

Das Cholesterin ist in der Milch in Form von Cholesterinfettsäureestern vorhanden. Aus der Tatsache, daß in der Magermilch ein verhältnismäßig hoher Cholesteringehalt festgestellt wurde, wird geschlossen, $\mathrm{daB}$ in der Milch ein Teil des Cholesterins kolloid in der Molke vorhanden ist, ein anderer Teil mit dem Fett zusammen als fettlöslicher. Bestandteil vorkommt.

Außer dem Cholesterin konnte im Milchfett eine weitere, unverseifbare alkohol- und ätherlösliche „Begleitsubstanz" festgestellt werden.

\section{Literaturverzeichnis.}

1) Winda us, Zeitschr. f. physiol. Chemie 65. 1910. - ${ }^{2}$ ) Langstein - Me yer, Säuglingsernährung und Säuglingsstoffwechsel. Wiesbaden 1914. S. 48. - ${ }^{3}$ ) Handbuch der Biochemie des Menschen und der Tiere (O p penhei mer) III, 1, S. 396. - $\left.{ }^{4}\right)$ Biochemisches Handlexikon Bd. III S. 204. - 5) Tolmatscheff, HoppeSeylers med. -chem. Untersuchungen. Berlin 1867, S. 272. -6) A. Bö mer, Zeitschr. f. Unters. d. Nahrungsmittel 1, 81. 1898. - 7) A. Kirsten, Zeitschr. f. Unters. d. Nahrungsmittel 5, 833. 1902. - $\left.{ }^{8}\right)$ Schmidt-Mülheim, Arch. f. d. ges. Physiol. 28, 217. 1882. - $\left.{ }^{\ominus}\right)$ Beu mer, Monatssehr. f. Kinderbeilk. 15, 581. 1919. - 10) Fränkel, Wiener klin. Wochenschr. 1913, S. 2198. - $\left.{ }^{11}\right)$ Rosenthal, Deutsche med. Wochenschr. 1919, Nr. 21, S. 571.

Wacker, München, Reisingstr. 13. Beck, München, Bavariaring 10. 